\title{
Gender Inequality and Its Effects in Females Torture Survivors
}

\author{
Ibrahim Kira ${ }^{1}$, Jeffery Ashby ${ }^{2}$, Linda Lewandowski ${ }^{3}$, Iris Smith ${ }^{4}$, Lydia Odenat ${ }^{4}$ \\ ${ }^{1}$ Center for Cumulative Trauma Studies, Stone Mountain, USA \\ ${ }^{2}$ Georgia State University, Atlanta, USA \\ ${ }^{3}$ Wayne State University, Detroit, USA \\ ${ }^{4}$ Emory University, Atlanta, USA \\ Email: kiraaref@aol.com
}

Received January $31^{\text {st }}, 2012$; revised February $23^{\text {rd }}, 2012$; accepted March $27^{\text {th }}, 2012$

\begin{abstract}
The study explores the effects of gender discrimination GD as type III trauma in 359, (160 females and 199 males) torture survivors. Data includes measures of GD and other traumas, PTSD and cumulative trauma disorders CTD. GD found to decrease PTSD symptoms in males favoring mental health status of males, and increase CTD symptoms in females. GD mediated the effects of personal identity traumas on PTSD and CTD symptoms of psychosis/dissociation; executive function deficits, and suicidality. The results highlight GD as type III trauma that contributes to the mental health differences between males and females.
\end{abstract}

Keywords: Gender Discrimination; Torture Survivors; PTSD; Cumulative Trauma Disorders; Type III Trauma

\section{Introduction}

\section{Torture and Gender Inequality: A Feminist Perspective}

The adverse effects of gender discrimination on the mental health outcomes of women have been a well-documented phenomenon in the research milieu. Gender discrimination (GD), defined as the assignment of values to real or imagined differences between genders, remains a ubiquitous part of the female experience and a major mental health concern for women worldwide. GD has been attributed to a number of poor mental health outcomes, including psychological distress (Dambrun, e. Defined as the intentional infliction of psych2007), low selfesteem (Schmitt, Branscombe, \& Postmes, 2003), anger (Swim, Hyers, Cohen, \& Ferguson, 2001), low self-efficacy, and depression and anxiety symptoms (Landrine, Klonoff, Gibbs, Manning, \& Lund, 1995). Despite the abundance of research on the etiology and consequences of GD, there remains a dearth of literature on the impact of this societal ill on the psychological well-being of female refugee torture survivors.

Research on GD has provided some insight into its deleterious effects on economic, political, and educational institutions in global communities. According to a qualitative focus group study of African female refugee torture survivors (conducted by the authors), GD, as well as the promotion of extreme male dominance and asymmetrical patriarchal world views, was perceived to be instrumental in the development of conditions/cultural environments that foster torture and other human rights abuses. Study participants argued that gender inequality gave way to the emergence of dominant male dictators, military junta, tribalism, and political, military and intelligence institutions that promote torture, oppression, discrimination and human rights violations against men and women alik ological, physical, or emotional pain or deprivation, torture is a practice commonly used in an astonishing array of societies for purposes of punishment, intimidation, and coercion (Pope, 2001).
Research on the experiences of torture survivors has found it to be significantly predictive of post-traumatic stress, depression, anxiety, and somatic complaints (Punamaki, Qouta, \& Sarraj, 2010). The primary aim of the present investigation is to explore how GD affects the mental health of female refugee torture survivors.

\section{Gender Discrimination GD as Type III Identity Trauma}

Traumatology developmental theorists have identified GD as a unique form of trauma that can have profound and negative mental health effects on clients (Kira, 2001; Kira et al., 2008). As such, a trauma taxonomy has been proposed to classify various forms of trauma based on their severity and complexity. According to this classification system, Type I trauma consists of a singular and potentially traumatic event (e.g. car accident), while Type II trauma is the complex and potentially repeated trauma that is discontinued (e.g., sexual or physical abuse). Type III represents ongoing social structural violence that represents mostly inter-group traumas that are without a foreseeable end (e.g., poverty, racism, discrimination, including GD), and Type IV are the multiple concurrent or sequential traumas occurring across the lifespan that have potential cumulative effects. Among the four types, traumatologists have identified Type III traumas as potentially the most serious kind, in terms of their impending adverse effects on the individual (Kira et al., 2008). It is important to note that the accumulative kindling dynamics are present in both Type III and IV traumas. It is argued that Type III trauma, due to its ongoing nature, may mediate or moderate the effects of other trauma types. Additionally, Type III is potentially the most serious kind, in terms of its negative effects on the individual due to its duration and lasting impact (Kira et al., 2008). Research has also demonstrated that Type III trauma tends to be internalized by those who employ this defense mechanism to cope with continuous 
exposure to painful micro- and macro-aggressions. Such acceptance of discrimination and stereotypes can harm self concept and efficacy and sense of control which are keys to health and mental health. However, some others resist the discrimination at different levels (e.g., mild, moderate, or tough resistance).

The aforementioned trauma types have been found to directly and indirectly impair emotional processing and cognitive functioning. The accumulation, kindling, and amplification dynamics makes cumulative trauma effects significantly different from a single Type I or Type II complex trauma, in that the effects of one trauma cannot be isolated from the other several traumas that the same individual has endured before or after its occurrence. The additive effects of multiple traumas amplify the severity of trauma related mental health symptoms and may be significantly related to executive function deficits and life achievements. Cumulative dynamics of GD's related microand macro-traumatic stressors across life span of women can be at play. The effects of cumulative dynamics of GD events can be severe beyond the PTSD syndrome. Cumulative trauma and poly-victimization across the life span have been found to contribute to significant unique variance in mental health outcomes beyond that accounted for by the combination of all aggregate trauma and victimization types (Kira, et al., 2008a; Richmond et al., 2009).

\section{Dimensions and Dynamics of Gender Discrimination GD}

Patriarchal systems have been identified as the foundation of women's subordination and positioning as second-class citizens in most of the contemporary cultures (Hunnicutt, 2009; Walby, 1990; Yllo, 1993). Childhood socialization, by family and social institutions, is one of the primary methods through which patriarchal values and gender expectations shape core dimensions of the self/gender identity. The core concept of patriarchy - systems of male domination and female subordinationevokes images of gender hierarchies, dominance, and sometimes power struggle (e.g., Brownmiller, 1975; Walby, 1990; Yllo, 1993; Hunnicutt, 2009). Patriarchy and institutionalized gender stratification shapes power structures, in turn creating GD that determines differential access to economic and social resources, as well as the perception of personal and collective self.

GD may include micro and macro, implicit and explicit gender related discriminative aggressive events that may include bullying, coercive control and violence against girls and women over a lifetime. Such aggressions often begin in childhood, occur concurrently, sequentially, or over the course of lifetime, and come from individuals, families and institutions. Internalization of comparative degraded status does not cancel the negative effect of such life term gender focused cumulative aggressions and degradations.

Gender-based violence (GBV) serves to maintain an unequal balance of power between men and women. GBV is a risk factor for injury and disability; executive function deficits, e.g., inattention, mental health disorders; chronic pain syndromes, somatic complaints; and other negative health behaviors (smoking, alcohol and drug abuse, physical inactivity, overeating) for women (Watts \& Zimmerman, 2002; Zimmerman et al., 2003). In domestic violence, women are usually the victims of the attack and the perpetrator may well be motivated directly by the desire to demonstrate his own dominance to enforce male power and control over women (Heise, 1998).

$\mathrm{GD}$, as manifested in most cultures, tends to validate, favor, empower and strengthen male's feelings of control and position as a dominant agent in the family and society. In turn, it suppresses female's personal identity, increases stereotype and injustice against them by the family and by social institutions, and gives way to relative feelings of powerlessness, loss of perceived control, decreased collective (gender) and personal self-esteem, self-efficacy, and agency, which eventually lead to diminished mental health potential (Swim, Hyers, Cohen, \& Ferguson, 2001).

GD against girls by parents during childhood initiates, reinforces, and cross-generationally perpetuates the practice of unequal treatment of females over the lifetime. Such types of traumas, because they are entrenched in the structure within the family and society, tend to be internalized and accepted by the victim as the normal course of life (Heise, 1998); however, internalizing the ongoing traumas does not necessarily cancel their negative effects on identity development, the concept of self and on physical and mental health of the victim. Suppressing or reframing thoughts and emotions through internalization, accepting culturally endorsed rationalization and submission to a second class status may even create violated self and induce degraded self-worth.

GD, that subjugates females, can arouse, sensitize, and bias females to be more prone to over respond to Type I and Type II stressors, as well as mediate or moderate their effects. Women may experience fewer other traumas than men, however GD, as previous and ongoing trauma, continue to sensitize them to stress, yielding more internalizing and more severe symptoms, in general, compared to men who do not suffer such GD (Emslie et al., 2002; Goldberg \& Williams, 1988; Macintyre, Ford, \& Hunt, 1999, Astbury, 2006; Dambrun, 2007; Rosenfield, 1999; WHO, 2006). On the other hand, GD that favors male dominant actors, does not only make them less vulnerable, but also empowers them to act aggressively and display more externalizing symptoms (e.g., Mejia, 2005, Scott, 1998; Hawton et al., 2002; Parker \& Roy, 2001; Linzer et al., 1996). Overall, GD may be lead to losses for both genders when mental health internalizing/externalizing outcomes are taken into consideration.

Further, GD overlaps, for females, with the other Type III traumas (e.g., racism, stigma, poverty, discriminations and other forms of social structural violence), producing different cumulative traumagenic dynamics that predispose the affected individual to respond differently to subsequent stressors. GD intersects with other discriminations enforced by social structural violence against women and minorities, (e.g., race, minority status) adding to the negative effects of its cumulative dynamics (Pittaway, 1999; Pittaway \& Bartolomei, 2001).

While GD perpetuates micro and macro aggressions against females, women may engage in systemic violence, for example prostitution, as a way of resisting and negatively responding to the social structural violence of gender victimization (Wesley, 2006). Research indicates that GD is negatively correlated with distributive and procedural justice, and positively linked to work conflict (Foley et al., 2005; Gutek, Cohen, \& Tsui, 1996). It also showed a negative correlation with job satisfaction and organizational commitment and a positive correlation with intentions to leave (Foley et al., 2005). Perceived procedural injustice has been positively linked to retaliation against the organization (Skarlicki \& Folger, 1997); perceived distributive 
injustice has been positively linked to employee theft (Greenberg, 1990);

Some studies seem to suggest that GD contributes to the documented differences in mental health between males and females. Klonoff, Landrine, \& Campbell (2000) found that women who experienced frequent sexism had significantly more depressive, anxious, and somatic symptoms than men, whereas women who experienced little sexism did not differ from men on any symptom measure. They found that negative sex stereotyping, isolation, and sexual objectification was associated with mental heath symptoms such as depression, anxiety, somatization and low self esteem (Klonoff, Landrine, \& Campbell, 2000). Findings suggest that gender discrimination may account for such gender differences in psychiatric symptoms (Landrine et al., 1995). Most studies found a significant relationship between gender and different mental health variables explaining between $5 \%$ and $15 \%$ of the observed variance. According to Dambrun (2007), perceived personal gender discrimination mediates the relationship between gender differences in mental health. Berg (2006) found that the most predicttive variable for females' trauma was recent sexist degradation, accounting for $20 \%$ of the variance in PTSD scores.

The purpose of the present study is to examine the potential role of GD in the development of cumulative trauma disorders (CTD) and symptoms of PTSD among female refugee torture survivors.

\section{Hypotheses}

Hypothesis 1: GD for females by parents (GD-P) and by society (GD-S) has significant negative effects on their mental health and executive functions.

Hypothesis 2: GD is protective factor for males that lead to decreased PTSD symptoms for them, while it is a risk factor for females that lead to increased symptoms for them.

Hypothesis 3: GD, as ongoing life-long term type III trauma sensitizes females to other life time type I and type II traumas, and mediates and/or moderates the effects of such traumas (e.g., personal identity, collective identity, survival and secondary traumas) on CTD and PTSD.

\section{Methods}

\section{Participants}

Participants are 359 primary and secondary torture survivors (a primary torture survivor is the person that had been subjected directly to torture, while a secondary torture survivor is one of his/her close family members). The sample for this study consisted of all the clients in the CTTS data base that were seen and screened in the Center between April 2008 and the end of September 2009. There were 160 females and 199 males seen during this time period. The ages of participants ranged from 12 to 79 years. The participants include 215 primary torture survivors and 143 secondary torture survivors (family members). For the females that most of our analysis will focus on, there are 53 primary torture survivors and 107 secondary torture survivors (affected family members). The participants came from 32 countries with the majority from Iraq $(n=99$, Female $=48$, Male $=51)$, Burma $(\mathrm{n}=93$, Female $=31$, Male $=62)$, Bhutan $(\mathrm{n}=77$, Female $=42$, Male $=35)$, Somalia $(\mathrm{n}=31$, Female $=21$, Male $=10)$, and others $($ Female $=21$, Male $=38)$. Others include refugees' torture survivors from, Afghanistan,
Chad, Congo, Cuba, Eritrea, India, Iran, Liberia, Nigeria, Russia, Rwanda, Zimbabwe, China and others. Female participants' employment, in their own countries, included: farmer $=39$, teacher $=16$, house wife $=15$, student $=12$, business women $=$ 9 , lab technician and medical assistant $=4$, seller $=7$, engineer $=1$, other occupations $=57$. Fourteen percent $(14 \%)$ of females were $12-19,36 \% 20-35,43 \% 36-55$, and $7 \%$ were $56-79$ years old. The majority (55.8\%) were married, $1.3 \%$ living with partners, $19.9 \%$ single and never married, $3.8 \%$ divorced, $12.8 \%$ are widows, $6.4 \%$ had either a missing spouse or a spouse that still resided in their home country. Average numbers of children for those who are or were married are 5.6. Most of them are new arrivals within 2 - 6 month of entry (95\%), few (less than $5 \%$ ) have been in US more than a year. GD is most profoundly observed in low-income economies of most of refugee cultures. (e.g., World Health Organization, 1988., Christiana \& Okojie 1994, Glick \& Fiske, 1996, Heise, Pitanguy, \& Germain, 1998).

\section{Measures}

PTSD Measure-(CAPS-2) (18 items): This measure was developed by Blake et al. (1990) and is widely used to assess post-traumatic stress disorder (PTSD). It is a structured clinical interview that assesses 17 symptoms rated on frequency and severity on a 5-point scale. CAPS demonstrated high reliability with a range from $0.92-0.99$ and showed good convergent and discriminant validity (Weathers, Keane, \& Davidson, 2001). The measure utilized in adult and adolescents samples. In this study, we used the frequency sub-scale of CAPS-2 that is currently widely used in psychiatric literature. It has, in this mixed sample, Cronbach alpha reliability coefficient of .94 for all participants, which indicates a good reliability. The scale has four sub-scales: re-experiencing, avoidance, arousal and emotional numbness/dissociation. Reliability of the four sub-scales in our sample are adequate to high (alphas are $.96, .92, .89$ and .85 respectively). Further the alpha coefficients were high across all national origin groups (Bhutanese $=.92$, Burmese $=$ 91 , Iraqi $=.85$, Somali $=.96$, others $=.97$. The measure reliabilities were high in each national origin female groups as well (Bhutanese $=.89$, Burmese $=.93$, Iraqi $=84$, Somali $=.96$, and all others $=.96)$.

Cumulative Trauma Disorders Measure CTD (15 items). The measure has been developed on five community and clinic samples of adults and adolescent Iraqi refugees, Arab Americans, and African Americans. It is an index measure that covers 13 different symptoms: depression, anxiety, somatization, dissociation, auditory and visual hallucinations, avoidance of being with people, paranoid ideations, concentration and memory deficits, loss of self control, feeling too harsh with family and with people in general, feeling suicidal, and feeling like hurting self. Exploratory factor analysis found four factors: Executive function deficits, suicidality, psychosis/dissociation, and depression/anxiety interface. Confirmatory factor analysis confirmed this structure. It has good reliability (ranged from .85 and .98). Test-retest reliability in a 6 week-interval is .76. The measure has good predictive validity. Different kinds of traumas, and cumulative trauma in general accounted for signifycant variance as predictors of CTD symptoms (Kira, 2004; Kira, Clifford, Wiencek, \& Al-haider, 2001, Kira, Clifford, \& AlHaider, 2002, 2003; see also Kira et al., 2006, 2007). The measure was found to be highly correlated with PTSD, DASS- 
A anxiety and CES-D depression measures in a clinic sample (n = 399) which substantiate its convergent validity. It was found to be highly negatively correlated with futuristic orientation, socio-cultural adjustment and post-traumatic growth which adequately substantiate its divergent validity. It has, in this mixed sample Cronbach alpha reliability coefficient of .98 , which indicates a good reliability. Reliability of the four subscales in the current study was found to be high $(.95, .97, .98$, and .96 respectively). Further the alpha coefficients were high across all national origin groups (Bhutanese $=.93$, Burmese $=.94$, Iraqi $=.94$, Somali $=.89$, others $=.94$. The measure reliabilities were high in each national origin female groups as well (Bhutanese $=.92$, Burmese $=.95$, Iraqi $=93$, Somali $=.92$, and all others $=.87$ ).

CTS Cumulative Trauma Scale (33 items) short form: CTS screens for the occurrence and frequency of trauma across life time. The measure is short form of a longer version that utilized taxonomy of traumas that are based on child and adult developmental theories. It was validated previously in Iraqi refugees and found to have good reliability, construct, divergent, convergent, and predicative validity (Kira et al., 2008a, 2008b; Kira et al., 2011). The measure originally has six main categorical sub-scales (attachment, for example abandonment by mother, personal identity, for example sexual abuse or rape, collective identity, for example oppression, and family, seconddary, and survival traumas). Different sub-categories were further added, e.g., gender discrimination, and torture. The total score represents the cumulative trauma load that the individual endured across life span. For the purpose of this study we focused on cumulative trauma occurrence for the total scale and for other six trauma types. The six trauma types include: personal identity traumas, e.g. sexual abuse, physical abuse, rape, robed or mugged, collective identity traumas, e.g., oppression, discrimination, survival traumas, e.g., shot at or stabbed, secondary trauma, e.g., witnessing or hearing about others traumas, torture trauma, and gender discrimination trauma. The measure has, in this mixed sample an adequate Cronbach alpha reliability coefficient of .81 . Alpha reliabilities for the sub-scales in the present data are as follows: Torture $=.89$ ( 2 items scale), gender discrimination, GD $(2$ items scale $)=.62$, survival trauma $(3$ items scale $)=.60$, secondary traumas $(3$ items scale $)=.66$, personal identity traumas $(15$ items scale $)=.62$, and collective identity traumas (6 items scale) $=.68$, gender discrimination GD in Iraqi females was .80 , and .65 for the others. Such reliability coefficients are acceptable for short scales with binary response questions. GD sub-scale consists of two items one asks about the occurrence of gender discrimination by parents and the second asks about the gender discrimination by other society members and institutions. Because we used each item as a separate measure one for GD by parents (GD-P) and the other for GD by society (GD-S), in our analysis, we calculated the reliability of each. Following the Wanous and Hudy (2001) method of estimating single-item reliability, we conducted factor analysis of the CT measure, the reliability of GD-P single item scale ranged between .69 (communality of the item) and .76 (factor loading). The reliability of GD-S scale ranged between .67 (communality of the item) and .72 (factor loading).

Further the alpha coefficients for CTS (the total scale) were adequate across all national origin groups (Bhutanese $=.86$, Iraqi $=.80$, Somali $=.70$, others $=.80$ ), except for Burmese (.50), which is considered relatively low. The measure reliabilities were adequate in each national origin female groups as well (Bhutanese $=.69$, Iraqis $=79$, Somalis $=.75$, and all others $=.79)$.

\section{Procedures}

Study investigators utilized an existing data set from a clinical database developed by a Center for Torture and Trauma Survivors (CTTS) that includes mental health data collected for all its clients. The data include a comprehensive intake conducted by qualified staff and mental health screening that included measures for PTSD- Clinician-Administered Posttraumatic Stress Scale CAPS-2, CTD and different trauma occurrence including gender discrimination by parents and society. The procedures in the clinic meet all HIPPA regulations concerning clients' protection. The assessments were conducted through face to face interviews in the clinic. Participants were referred to clinic by resettlement agencies and health screening authorities as a torture victims.

\section{Data Analysis}

Study investigators explored the trauma profiles for each gender using two-ways cluster analysis, and the mental health differences based on the two trauma profiles. Correlational analysis was conducted between GD sub-scale, its two items, and PTSD and CTD and their sub-scales in the females subsample $(\mathrm{N}=160)$. Multiple regression analysis was conducted with PTSD and CTD as dependent variables and other traumas including GD as independent variables. Different plausible path models were tested for direct, indirect effects, using structural equation model SEM (AMOS 7 software), (Arbuckle, 2006). Model fit indices were selected in accordance with several recommendations and included the normed $\chi^{2}$ test statistic $\left(\chi^{2} / d f\right)$, the root mean square error of approximation (RMSEA) and the comparative fit index (CFI). $\chi^{2} / d f$ values $<5.0$ are considered acceptable; RMSEA values $\leq .05$ indicate close fit, values .05 to .08 indicate reasonable fit, and values $>.10$ indicate poor fit. CFI values $>.95$ indicate good fit (e.g., Kline, 2005; Hu \& Bentler, 1999). Bootstrap ( $\mathrm{N}=200$ ) with biascorrected confidence intervals was used to test the significance of the direct and indirect effects of each variable in the model. Bootstrapping is a computer-intensive re-sampling technique. This procedure involves generating bootstrap samples based on the original observations. Bootstrapping is often used to get a better approximation of sampling distribution of a statistic than its theoretical distribution provides, especially when assumption of normality may be violated. Bootstrapping is more robust modern statistics that are used to generate and to create a sampling distribution, and bootstrapped distribution is used to compute $p$ values, test hypotheses and generate confidence intervals for direct and indirect effects (e.g., Erceg-Hurn \& Mirosevich, 2008).

\section{Results}

\section{Hypothesis 1 and 2: The Effects of GD, GD-P and GD-S}

The differences in gender discrimination between the major nationalities in the current sample were not statistically significant. Correlational relationships were examined between gender discrimination scale (total), gender discrimination by parents, gender discrimination by society, PTSD and its four sub- 
scales, and CTD and its four sub-scales in females sub-sample. GD (Total) was found to be significantly correlated with PTSD, CTD and all their sub-scales with the highest correlation with CTD-psychosis sub-scale $(r=.42)$. GD-P was not significantly correlated with PTSD; however it was significantly correlated with PTSD-Arousal sub-scale and significantly correlated with CTD, CTD-executive functions deficits, suicidality, psychosis/ dissociation sub-scales. PGD-S (GD by society) has the highest significant correlations with PTSD, CTD and all their subscales. Table 1 presents these results.

Separate multiple regression analyses was conducted for males and females, with PTSD and CTD alternatively as dependent variables and other trauma types including GD as independent variables. While other traumas generally predicted increase in PTSD and CTD, GD predicted significant decrease in PTSD in males but not in females, and significant increase in CTD in females but not in males. Tables $\mathbf{2}$ and $\mathbf{3}$ present these results.

Table 1.

Pearson correlations: Associations of Gender Discrimination for females all ethnic backgrounds with PTSD and CTD and components. N $=160$.

\begin{tabular}{llll}
\hline & GD-Total & GD-P & GD-S \\
\hline PTSD & $.15^{*}$ & .07 & $.17^{*}$ \\
PTSD-Experiencing & $.23^{* *}$ & $.15^{*}$ & $.23^{* *}$ \\
PTSD-Arousal & $.32^{* *}$ & $.20^{*}$ & $.32^{* *}$ \\
PTSD-Avoidance & $.29^{* *}$ & $.19^{*}$ & $.28^{* *}$ \\
PTSD-Numbness/Dissociation & $.16^{*}$ & .04 & $.21^{* *}$ \\
CTD & $.38^{* *}$ & $.26^{* *}$ & $.37^{* *}$ \\
CTD-Depression/Anxiety & $.20^{*}$ & .12 & $.21^{* *}$ \\
CTD-Psychotic/Dissociation & $.35^{* *}$ & $.23^{* *}$ & $.34^{* *}$ \\
CTD-Executive functions deficits & $.32^{* *}$ & $.22^{* *}$ & $.30^{* *}$ \\
CTD-Suicidality & $.34^{* *}$ & $.26^{* *}$ & $.30^{* *}$ \\
CTD-Neuroticism & $.27^{* *}$ & $.17^{*}$ & $.27^{* *}$ \\
CTD-Psychoticism & $.42^{* *}$ & $.28^{* *}$ & $.41^{* *}$ \\
\hline
\end{tabular}

Note: GD-Total $=$ Gender discrimination scale, GD-P $=$ Gender discrimination by parents, GD-S $=$ Gender Discrimination by society. ${ }^{+}$Close to significant, (at .10 level). ${ }^{* *}$ Correlation is significant at the 0.01 level (2-tailed). ${ }^{*}$ Correlation is significant at the 0.05 level (2-tailed).

Table 2.

Multiple regression for the effects of traumas and GD on PTSD.

\begin{tabular}{|c|c|c|c|c|c|c|}
\hline \multirow{2}{*}{ Independent variables } & \multicolumn{3}{|c|}{ Males } & \multicolumn{3}{|c|}{ Females } \\
\hline & B & SE & Beta & B & $\mathrm{SE}$ & Beta \\
\hline Secondary Traumas & 4.71 & 1.22 & $.32^{* *}$ & 4.57 & 1.28 & $.29^{* *}$ \\
\hline $\begin{array}{c}\text { Gender } \\
\text { Discrimination }\end{array}$ & -13.96 & 6.92 & $-.14^{*}$ & -1.38 & 5.03 & -.02 \\
\hline $\begin{array}{l}\text { Collective Identity } \\
\text { Traumas }\end{array}$ & .92 & .54 & $.11+$ & .54 & .65 & .06 \\
\hline $\begin{array}{c}\text { Personal } \\
\text { Identity Traumas }\end{array}$ & 3.20 & .704 & $.40^{* *}$ & 3.81 & .74 & $.43^{* *}$ \\
\hline
\end{tabular}

Note: ${ }^{+}$Close to significant, (at .10 level). ${ }^{* *}$ Correlation is significant at the .01 level (2-tailed). "Correlation is significant at the .05 level (2-tailed).
Table 3.

Multiple regression for the effects of traumas and GD on CTD.

\begin{tabular}{lcccccc}
\hline & \multicolumn{3}{c}{ Males } & \multicolumn{3}{c}{ Females } \\
\cline { 2 - 7 } \multicolumn{1}{l}{ Independent variables } & $\mathrm{B}$ & SE & Beta & B & SE & Beta \\
\cline { 2 - 7 } & 1.42 & .87 & $.15^{+}$ & 3.00 & .74 & $.31^{* *}$ \\
$\begin{array}{l}\text { Secondary } \\
\text { Traumas }\end{array}$ & & & & & & \\
$\begin{array}{l}\text { Gender } \\
\text { Discrimination }\end{array}$ & -4.58 & 5.07 & -.07 & 9.67 & 2.62 & $.27^{* *}$ \\
$\begin{array}{l}\text { Collective } \\
\text { Identity Traumas }\end{array}$ & .70 & .38 & $.13+$ & -.22 & .39 & -.04 \\
$\begin{array}{l}\text { Personal } \\
\text { Identity Traumas }\end{array}$ & 1.70 & .52 & $.33^{* *}$ & 1.49 & .43 & $.28^{* *}$ \\
\hline
\end{tabular}

Note: ${ }^{+}$Close to significant, (at .10 level). ${ }^{* *}$ Correlation is significant at the .01 level (2-tailed). ${ }^{*}$ Correlation is significant at the .05 level (2-tailed).

\section{Hypothesis 3 (Sensitization, Mediation and Moderation Hypothesis)}

Exploring the trauma profile for each gender using two way cluster analysis, findings suggest that males, in this population, are significantly higher in total trauma load as compared to females (11.25 for males, and 9.68 for females). Females appeared to suffer more from torture, personal identity traumas, and survival traumas. There is no difference between them in collective identity or secondary traumas. The only trauma type that was significantly higher for females was gender discrimination. However, regardless of the higher trauma load in males, there is no gender differences in the severity levels of PTSD, or CTD (see Table 2). The only difference is that females have significantly higher scores in PTSD-hyperarousal sub-scale which may corroborate, to a degree, the sensitivity hypothesis. Such sensitivity is assumed to be related to the continuous subjection to gender discrimination that sensitizes them to differential arousal level in responding to other life stressors. Tables $\mathbf{4}$ and $\mathbf{5}$ present these findings.

To explore the GD mediation/moderation hypothesis, study investigators utilized SEM AMOS 7 software to build a model that reflects our theoretical assumptions and past research findings. Different plausible models were tested and all the models had adequate to excellent fit with the data. Among the two models presented, the first has all trauma types as independent variables, GD as mediating variable, and PTSD four factors (reexperiencing, arousal, avoidance and dissociation/numbness) as dependent variables. The model has good fit with the data (Chi Square $=11.078$, d.f. $=14, p=.680, C F I=1.000$, RMSEA $=.000)$. In this model, GD has direct effects on increased avoidance and arousal and indirect effects on increased re-experiencing. Personal identity traumas (PIT) have direct effects on increased perceived GD. GD mediated the PIT effects on increased avoidance, arousal and reexperiencing. Collective identity traumas (CIT) have direct effects on increased GD (close to significant). GD mediated CIT effects on increased avoidance. Survival traumas on the other side have significant negative effects on perceived GD. GD mediated the negative effects of survival traumas on decreased avoidance and arousal.

The second model has all trauma types as independent variables, GD as mediating variable, and CTD four factors (depresssion/anxiety interface, psychosis/dissociation, executive function deficits, and suicidality) as dependent variables. The model has good fit with the data (Chi Square $=9.301$, d.f. $=15$, 
Table 4.

Centroids that depict the trauma profiles for those that suffered perceived gender discrimination and those that do not in the sample $\mathrm{N}=359$.

\begin{tabular}{|c|c|c|c|c|c|c|c|c|c|c|c|c|}
\hline \multirow{2}{*}{$\begin{array}{c}\text { GD trauma clusters } \\
\text { Experienced GD }\end{array}$} & \multicolumn{2}{|c|}{ Cumulative trauma load } & \multicolumn{2}{|c|}{ Torture } & \multicolumn{2}{|c|}{$\begin{array}{l}\text { Collective identity } \\
\text { traumas }\end{array}$} & \multicolumn{2}{|c|}{ Personal identity traumas } & \multicolumn{2}{|c|}{ Survival traumas } & \multicolumn{2}{|c|}{ Secondary trauma } \\
\hline & Mean & SD & Mean & SD & Mean & SD & Mean & SD & Mean & SD & Mean & SD \\
\hline 1. No & 7.97 & 2.70 & .82 & .95 & 3.55 & 1.70 & 2.15 & 1.05 & .68 & .69 & .72 & .78 \\
\hline 2. Yes & 14.68 & 3.72 & 1.67 & .65 & 3.83 & 2.04 & 5.26 & 1.67 & 2.14 & .70 & 2.32 & .77 \\
\hline Combined & 10.55 & 4.52 & 1.14 & .94 & 3.66 & 1.84 & 3.35 & 2.01 & 1.24 & .99 & 1.34 & 1.10 \\
\hline
\end{tabular}

Table 5.

The differences between genders in trauma types, PTSD, CTD and their sub-scales.

\begin{tabular}{|c|c|c|c|c|c|c|c|}
\hline & Gender: & $\mathrm{N}$ & Mean & SD & SE & Mean Difference & $\mathrm{t}$ \\
\hline \multirow{2}{*}{ Torture Traumas } & Males & 199 & 1.50 & .75 & .05 & \multirow{2}{*}{.85} & \multirow{2}{*}{$9.97^{* * *}$} \\
\hline & Females & 160 & .64 & .88 & .07 & & \\
\hline \multirow{2}{*}{ Survival Traumas } & Males & 199 & 1.46 & .89 & .06 & \multirow{2}{*}{.50} & \multirow{2}{*}{$5.0^{* * *}$} \\
\hline & Females & 160 & .96 & .99 & .08 & & \\
\hline \multirow{2}{*}{ Secondary Traumas } & Males & 199 & 1.40 & 1.07 & .08 & \multirow{2}{*}{.14} & \multirow{2}{*}{1.22} \\
\hline & Females & 160 & 1.27 & 1.09 & .09 & & \\
\hline \multirow{2}{*}{ Collective Identity Traumas } & Males & 199 & 3.67 & 1.78 & .13 & \multirow{2}{*}{.01} & \multirow{2}{*}{.03} \\
\hline & Females & 160 & 3.67 & 1.70 & .13 & & \\
\hline \multirow{2}{*}{ Personal Identity Traumas } & Males & 199 & 3.58 & 1.94 & .14 & \multirow{2}{*}{.47} & \multirow{2}{*}{$2.23^{* *}$} \\
\hline & Females & 160 & 3.11 & 2.01 & .164 & & \\
\hline \multirow{2}{*}{ Perceived Gender Discrimination } & Males & 199 & .01 & .16 & .01 & \multirow{2}{*}{-.05} & \multirow{2}{*}{$-1.98^{* *}$} \\
\hline & Females & 160 & .07 & .29 & .02 & & \\
\hline \multirow{2}{*}{ Cumulative Trauma Occurrence Scale } & Males & 199 & 11.25 & 3.88 & .28 & \multirow{2}{*}{1.57} & \multirow{2}{*}{$3.57^{* * *}$} \\
\hline & Females & 160 & 9.68 & 4.46 & .35 & & \\
\hline \multirow{2}{*}{ Post-Traumatic Stress Disorder (PTSD) } & Males & 199 & 14.70 & 15.65 & 1.11 & \multirow{2}{*}{-1.24} & \multirow[t]{2}{*}{-.739} \\
\hline & Females & 160 & 15.941 & 16.02 & 1.27 & & \\
\hline \multirow{2}{*}{ PTSD-Re-experiencieng Sub-Scale } & Males & 199 & 7.66 & 7.84 & .57 & \multirow[t]{2}{*}{-.05} & \multirow[t]{2}{*}{-.057} \\
\hline & Females & 160 & 7.71 & 7.39 & .60 & & \\
\hline \multirow{2}{*}{ PTSD-Avoidance Sub-Scale } & Males & 199 & 2.19 & 2.46 & .18 & -.001 & -.002 \\
\hline & Females & 160 & 2.19 & 2.68 & .22 & & \\
\hline PTSD-Arousal Sub-Scale & Males & 199 & 3.01 & 4.63 & .34 & -1.69 & $-2.94^{* *}$ \\
\hline & Females & 160 & 4.70 & 5.93 & .48 & & \\
\hline PTSD-Dissociation & Males & 199 & 1.93 & 3.50 & .25 & -10 & -263 \\
\hline 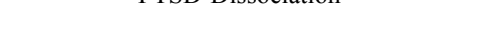 & Females & 160 & 2.03 & 3.51 & .28 & & \\
\hline Cumulative Trauma Disorders CTD & Males & 199 & 11.73 & 10.63 & .75 & -25 & -228 \\
\hline & Females & 160 & 11.98 & 10.18 & .81 & & \\
\hline CTD-Depression/Anxiety Interface & Males & 199 & 4.60 & 3.51 & .25 & -51 & -1325 \\
\hline & Females & 160 & 5.11 & 3.61 & .29 & & \\
\hline CTD-Fxecutive Functions Deficits Sub_Scale & Males & 199 & 1.91 & 2.13 & .15 & 11 & 474 \\
\hline 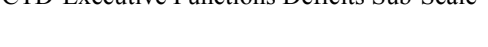 & Females & 160 & 1.80 & 2.04 & .16 & & \\
\hline CTD-Psychoticism /Dissociation Sub-Scale & Males & 199 & 1.60 & 2.52 & .18 & -14 & -527 \\
\hline & Females & 160 & 1.74 & 2.51 & .21 & & \\
\hline CTD_Suicidality Sub_Scale & Males & 199 & .75 & 1.44 & .10 & 04 & 278 \\
\hline & Females & 160 & .71 & 1.28 & .10 & & \\
\hline
\end{tabular}

Note: ${ }^{+} p<.10$ (close to significant) ${ }^{*} p<.05,{ }^{* *} p<.01,{ }^{* * *} p<.001$. 
$p=.861, C F I=1.000, R M S E A=.000)$. In this model, GD has direct significant effects on increased psychosis/dissociation and indirect effects on increased deficits in executive function and suicidality. PIT has direct effect on increased perceived GD. GD mediated their effects on increased on psychosis/dissociation, deficits in executive functions and increased suicidality. Collective identity traumas (CIT) have direct effects on increased GD (close to significant). GD mediated CIT effects on suicidality. Survival traumas on the other side have significant negative effects on perceived GD. GD mediated the negative effects of survival traumas on decreased psychosis/dissociation. GD does not seem to mediate secondary trauma effects in either model. Figures 1 and 2 present the direct paths for each model. Tables 6 and 7 include the decomposition of standardized direct, indirect, and total effects of the variables in each model.

\section{Discussion}

While sex differences in pro-social behavior that appear in research and match widely gender role beliefs, lie in the historical division of labor and has its origins in physical attributes and hormonal process of both genders (Wood \& Eagley, 2002), GD reflects another cultural dimension that exploits such differences to proclaim domination and privileges of male gender in patriarchal cultural and political systems (e.g., Walby, 1990). Such exploitation is gender specific identity trauma type III trauma that is ongoing and has accumulative effects and is potentially more severe in most refugee cultures.

GD tends to buffer against or decrease PTSD symptoms in males as the study findings indicated, favoring mental health status of males over females. GD is a protective factor for males and risk factor for females' mental health. GD, embedded in the culture, tends to validate, favor, empower and strengthen male's feelings of control and self esteem as a

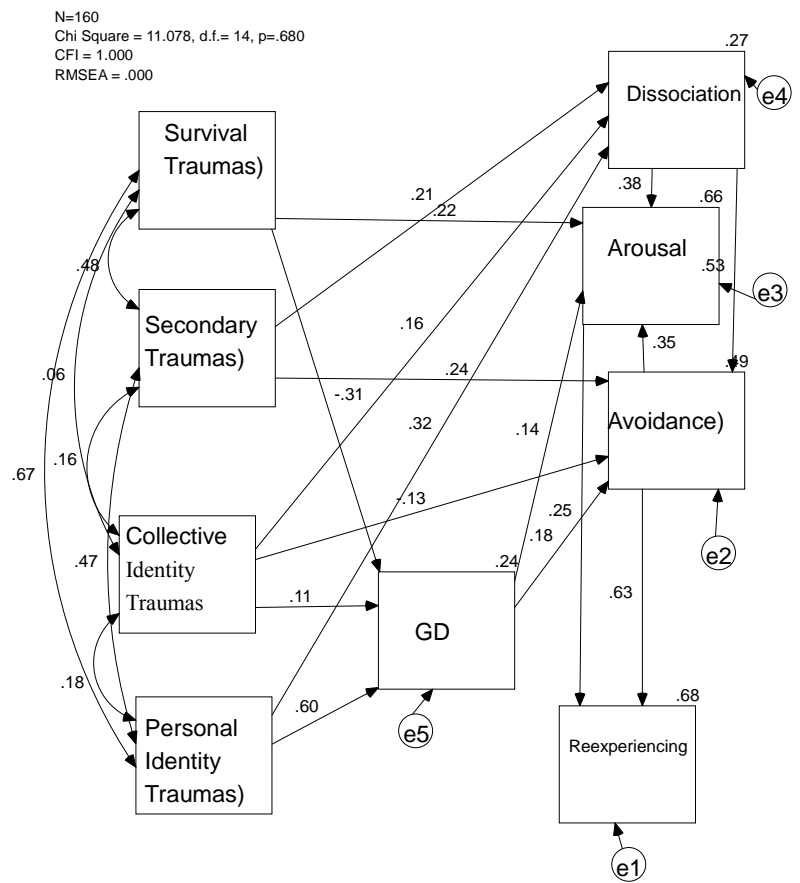

Figure 1.

Path model for the direct effects of different traumas on PTSD four components mediated by GD.

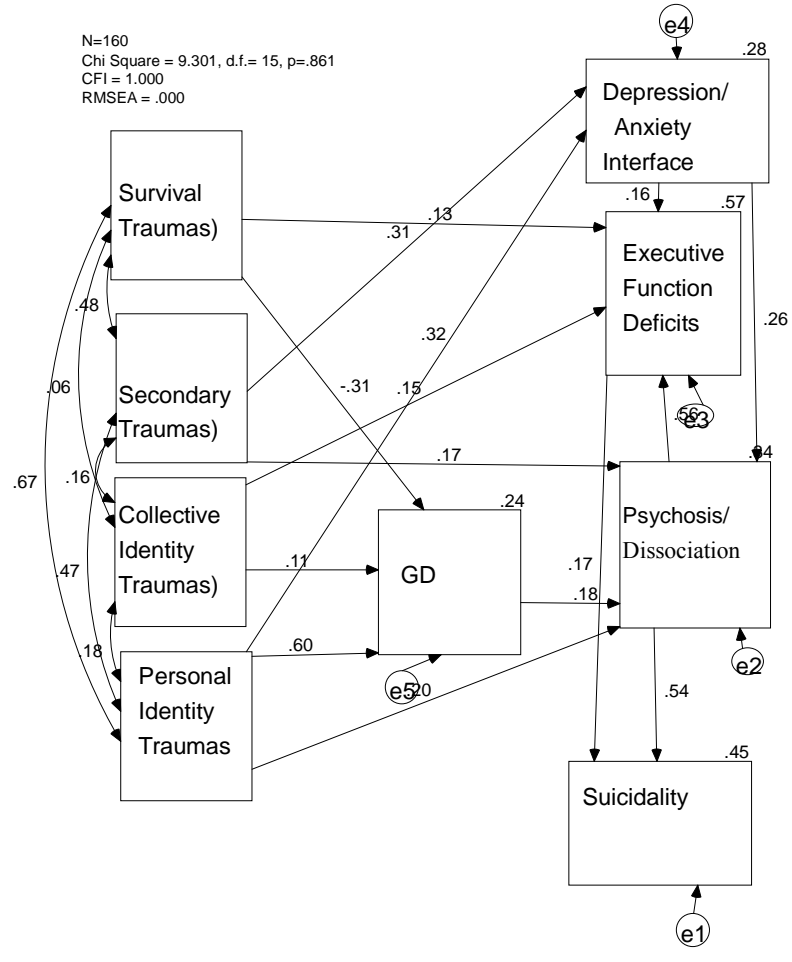

Figure 2.

Path model for the direct effects of different traumas on CTD four components mediated by GD.

dominant agent in the family and society, giving them such mental health advantage over females. On the other side, as the current study findings indicated, GD is associated with increase in the more complex symptoms of CTD in females and not males. GD suppresses female's personal gender identity, and increases stereotype, injustice and coercive control and potential violence against them by the family and by social institutions, creating relative feelings of powerlessness, loss of perceived control, decreased collective (gender) and personal selfesteem, self-efficacy, and agency (c.f., e.g., Swim, Hyers, Cohen, \& Ferguson, 2001).

The effects of gender discrimination by parents (GD-P) are worth noting. GD-P is associated, in females, with executive function deficits that include deficits in memory and of control of own reactions. It is associated with increase of suicidality, dissociation, psychotic reactions, avoidance and arousal. Neural mechanisms studies utilizing fMRI technology found that gender threat underlies women's underperformance in math (Krendl, Richeson, Kelley, \& Heatherton, 2008, see also Richeson et al., 2003). Other studies found no gender differences in genetic etiology in higher math performance in males (Petrill et al., 2009). Parenting style and family culture that favor males exerts its toll on the girls' executive functions, related potential achievements. Family gender discrimination while has serious effects, society's discrimination, especially, seems to have the most detrimental effects on all functioning of affected females. This speaks to pervasive societal-wide GD, which appears to account for most of the variance accounted for by family-specific GD.

The results of this study highlighted the serious effects of GD, as a complex ongoing serious trauma on females' mental 
Table 6.

Decomposition of standardized direct, indirect, and total effects of trauma variables on PTSD four components, and GD (a mediation model for gender discrimination).

\begin{tabular}{|c|c|c|c|c|c|}
\hline \multirow{2}{*}{ Causal Variables } & \multicolumn{5}{|c|}{ Endogenous Variables } \\
\hline & Dissociation/Numbness & GD & Avoidance & Arousal & Reexperiencing \\
\hline \multicolumn{6}{|l|}{ Personal Identity trauma } \\
\hline Direct Effects & $.321^{* *}$ & $.60^{*}$ & .000 & .000 & .000 \\
\hline Indirect Effects & .000 & .000 & $.28^{* *}$ & $.30^{* *}$ & $.25^{* *}$ \\
\hline Total Effects & $.32^{* *}$ & $.602^{*}$ & $.28^{* *}$ & $.30^{* *}$ & $.25^{* *}$ \\
\hline \multicolumn{6}{|l|}{ Collective Identity Traumas } \\
\hline Direct Effects & $.17^{*}$ & $.11+$ & $-.13^{*}$ & .000 & .000 \\
\hline Indirect Effects & .000 & .000 & $.108^{*}$ & .07 & .001 \\
\hline Total Effects & $.17^{*}$ & $.11+$ & -.03 & .07 & .001 \\
\hline \multicolumn{6}{|l|}{ Secondary Traumas } \\
\hline Direct Effects & $.21^{*}$ & .000 & $.24^{* *}$ & .000 & .000 \\
\hline Indirect Effects & .000 & .000 & $.11^{*}$ & $.21^{* *}$ & $.28^{* *}$ \\
\hline Total Effects & $.21^{*}$ & .000 & $.36^{* *}$ & $.21^{* *}$ & $.28^{* *}$ \\
\hline \multicolumn{6}{|l|}{ Survival Traumas } \\
\hline Direct Effects & .000 & $-.31^{*}$ & .000 & $.22^{* *}$ & .000 \\
\hline Indirect Effects & .000 & .000 & $-.06^{*}$ & $-.06^{*}$ & .003 \\
\hline Total Effects & .000 & $-.31^{*}$ & $-.06^{*}$ & $.15^{* *}$ & .003 \\
\hline \multicolumn{6}{|l|}{ Dissociation/ Emotional Numbness } \\
\hline Direct Effects & .000 & .000 & $.5^{3 * *}$ & $.39^{* *}$ & .000 \\
\hline Indirect Effects & .000 & .000 & .000 & $.19^{* *}$ & $.48^{* *}$ \\
\hline Total Effects & .000 & .000 & $.53^{* *}$ & $.57^{* *}$ & $.48^{* *}$ \\
\hline \multicolumn{6}{|l|}{ Gender Discrimination } \\
\hline Direct Effects & .000 & .000 & $.18^{*}$ & $.14^{*}$ & .000 \\
\hline Indirect Effects & .000 & .000 & .000 & $.06^{*}$ & $.17^{* *}$ \\
\hline Total Effects & .000 & .000 & $.18^{*}$ & $.20^{* *}$ & $.17^{* *}$ \\
\hline \multicolumn{6}{|l|}{ PTSD_Avoidance } \\
\hline Direct Effects & .000 & .000 & .000 & $.35^{* *}$ & $.63^{*}$ \\
\hline Indirect Effects & .000 & .000 & .000 & .000 & $.09^{* *}$ \\
\hline Total Effects & .000 & .000 & .000 & $.35^{* *}$ & $.72^{* *}$ \\
\hline \multicolumn{6}{|l|}{ Arousal } \\
\hline Direct Effects & .000 & .000 & .000 & .000 & $.25^{* *}$ \\
\hline Indirect Effects & .000 & .000 & .000 & .000 & 000 \\
\hline Total Effects & .000 & .000 & .000 & .000 & $.25^{* *}$ \\
\hline Squared Multiple Correlations & .27 & .24 & .49 & .66 & .68 \\
\hline
\end{tabular}

Note: ${ }^{+} p<.10$ (close to significant), ${ }^{*} p<.05,{ }^{* *} p<.01,{ }^{* * *} p<.001$. 
Table 7.

Decomposition of standardized direct, indirect, and total effects of trauma variables on PTSD, CTD and GD (a mediation model for gender discrimination).

\begin{tabular}{|c|c|c|c|c|c|}
\hline \multirow{2}{*}{ Causal Variables } & \multicolumn{5}{|c|}{ Endogenous Variables } \\
\hline & GD & Depression/Anxiety Interface & Psychosis/Dissociation & Executive Function Deficits & Suicidality \\
\hline \multicolumn{6}{|l|}{ Personal Identity trauma } \\
\hline Direct Effects & $.60^{*}$ & $.32^{* *}$ & $.20^{*}$ & .000 & .000 \\
\hline Indirect Effects & .000 & .000 & $.19^{* *}$ & $.27^{* *}$ & $.26^{* *}$ \\
\hline Total Effects & $.60^{*}$ & $.32^{* *}$ & $.39^{* *}$ & $.27^{* *}$ & $.26^{* *}$ \\
\hline \multicolumn{6}{|l|}{ Collective Identity Traumas } \\
\hline Direct Effects & $.11^{+}$ & .000 & .000 & $.15^{* *}$ & .000 \\
\hline Indirect Effects & .000 & .000 & $.02^{+}$ & $.01^{+}$ & $.04^{*}$ \\
\hline Total Effects & $.11^{+}$ & .000 & $.02^{+}$ & $.16^{* *}$ & $.04^{*}$ \\
\hline \multicolumn{6}{|l|}{ Secondary Traumas } \\
\hline Direct Effects & .000 & $.31^{*}$ & $.17^{*}$ & .000 & .000 \\
\hline Indirect Effects & .000 & .000 & $.08^{* *}$ & $.19^{* *}$ & $.17^{* *}$ \\
\hline Total Effects & .000 & $.31^{*}$ & $.25^{* *}$ & $.19^{* *}$ & $.17^{* *}$ \\
\hline \multicolumn{6}{|l|}{ Survival Traumas } \\
\hline Direct Effects & $-.31^{*}$ & .000 & .000 & $.13+$ & .000 \\
\hline Indirect Effects & .000 & .000 & $-.06^{*}$ & $-.03+$ & -.01 \\
\hline Total Effects & $-.31^{*}$ & .000 & $-.06^{*}$ & .10 & -.01 \\
\hline \multicolumn{6}{|l|}{ Gender Discrimination } \\
\hline Direct Effects & .000 & .000 & $.18^{* *}$ & .000 & .000 \\
\hline Indirect Effects & .000 & .000 & .000 & $.10^{* *}$ & $.12^{* *}$ \\
\hline Total Effects & .000 & .000 & $.18^{* *}$ & $.10^{* *}$ & $.12^{* *}$ \\
\hline \multicolumn{6}{|l|}{ Depression/Anxiety Interface } \\
\hline Direct Effects & .000 & .000 & $.26^{* *}$ & $.16^{* *}$ & .000 \\
\hline Indirect Effects & .000 & .000 & .000 & $.15^{* *}$ & $.19^{* *}$ \\
\hline Total Effects & .000 & .000 & $.26^{* *}$ & $.31^{* *}$ & $.19^{* *}$ \\
\hline \multicolumn{6}{|l|}{ Psychosis/Dissociation } \\
\hline Direct Effects & .000 & .000 & .000 & $.56^{* *}$ & $.54^{* *}$ \\
\hline Indirect Effects & .000 & .000 & .000 & .000 & $.10+$ \\
\hline Total Effects & .000 & .000 & .000 & $.56^{* *}$ & $.64^{* *}$ \\
\hline \multicolumn{6}{|l|}{ Executive Function Deficits } \\
\hline Direct Effects & .000 & .000 & .000 & .000 & .168 \\
\hline Indirect Effects & .000 & .000 & .000 & .000 & .000 \\
\hline Total Effects & .000 & .000 & .000 & .000 & .168 \\
\hline Squared Multiple Correlations & .24 & .29 & .34 & .57 & .45 \\
\hline
\end{tabular}

Note: ${ }^{+} p<.10$ (close to significant), ${ }^{*} p<.05,{ }^{* *} p<.01,{ }^{* * *} p<.001$. 
health. The study confirmed the hypothesis that GD, in females, have direct effects on increased PTSD arousal, avoidance, and reexperiencing symptoms, and increased CTD symptoms of dissociation/psychosis, executive function deficits, and suicidality.

GD seems to mediate the effects of some, but not all trauma types. GD mediated the specifically the effects of personal identity traumas (PIT) on increased PTSD symptoms of avoidance, arousal and reexperiencing and CTD symptoms of psychosis/dissociation; executive function deficits, and suicidality. GD mediated the effects of collective identity traumas (CIT) on increased PTSD avoidance and CTD suicidality. While PIT and to some extent CIT seems to accentuate perceived GD, survival traumas seems to mobilize women on survival issues and minimize their perceived GD. On the other hand, GD does not seem to mediate, in the current study, the effects of secondary traumas on PTSD or CTD symptoms.

The women in this sample reported experiencing significantly fewer traumas (by trauma count) than the male participants; however, they also reported higher PTSD symptoms. The only trauma in females' trauma profile that may explain such differences in their mental health is the high gender discrimination trauma that is continuous and cumulative, and mostly ignored as traumatic stressors for females. The ongoing implicit and explicit, micro and macro aggression, and gendered coercive control included in GD by family, by community and society, appears to have an effect on the women's reactivity and sensitivity to some other non-gendered traumatic events, especially those related to personal or collective identity. For women who have been tortured, the feelings of powerlessness and helplessness triggered by restrictive gender discrimination may lead to heightened negative responses to selective future traumatic events.

Various explanations have been proposed to explain gender difference in mental health (e.g., Astbury, 1999; BruchonSchweitzer, 2002; Jenkins, 1991), including biological, behaveioral, and social factors. In the present study, we examined the role of gender discrimination; a type III trauma that has accumulative effects, in great part, to the observed gender gap in psychological distress. This is at least a plausible alternative explanation of the differences between genders in mental health.

Gender discrimination, as ongoing internalized trauma, may continuously traumatize and sensitize females, thus leading them to have a heightened level of arousal and sensitivity in response to other traumas compared to their counterpart males that GD gave them advantage and relative protective buffer against further shorter term adversities.

Adopting a paradigm shift about the nature of GD as type III trauma that has been sometimes internalized, rationalized or resisted, will entail revising our legal standards for violence against women, as well as some of the assumptions and methodologies of gendered social sciences. This perspective reframes our understanding of some forms of domestic violence and gendered like crimes to be a liberty and human rights crimes rather than a crimes of assault. Seeing severe partner-perpetrated abuse, by a chauvinist male, as a human rights violation requires paradigm shift. The mechanisms of coercive control reveal political patterns in profoundly individual situations with the micro-regulation of everyday behaviors associated with stereotypic female roles (e.g., Stark, 2007). Our analysis goes even further, to reconsider, such gendered crimes of discrimination to be equal to other crimes of discrimination.
Some of such crimes can amount to be gender hate crimes and should be legally handled as such. Such paradigm shift would likely provide more effective state intervention into what were once considered private relationships and reduce or eliminate gender discrimination in society.

Another implication of our findings is the importance of reviewing our scientific perspective and research methods concerning gender. One of the cultural by-products of GD is the gendered science. Gendered science assumes that biological differences between genders underlie social and economic gender hegemony. Gendered science holds that genders are naturally unequal and therefore must be ranked hierarchically. It assumes that each gender has distinctive cultural behaviors and assigned roles linked to their biology (e.g., Bleier, 1984). Some psychologists contend that gender refers to biological characteristics of individuals whereas others assert that gender is a social construction that establishes and maintains a socio-political structural violence against women in societies and cultures. In research, researchers agree to use factitious gender categories as independent, predictor, or covariant variables in their theories and research designs. GD is usually lumped under one variable in analysis as gender variable. Gender category is static and limited conception of chunk and reflect a reification process, whereby dynamic and expansive processes are transformed into things or objects. Consolidating the components of Gender discrimination masks its traumatic and struggling dynamics and represents either inability to analyze and understand, or ignoring such dynamics by gendered science. The use of gender category as precise measure of some genuine psycho-logical theoretical construct accords scientific legitimacy to what are essentially gender stereotypes that psychologists share with the larger society (c.f., Bergvall, 1999). The methodologycal limitations of using gender category as independent variable make its replacement necessary. Utilizing a measure for GD or controlling for it, can be more scientifically helpful than just categorizing gender in research.

Advocating for a culture of gender equality and for cultural change toward this goal is important intervention to reduce or eliminate such gender gap in mental health. Gender equality may help reduce internalizing disorders in girls and women, and possibly externalizing disorders in boys and men. Further, if we accept feminist perspective on torture, discussed earlier, eliminating gender inequality world wide may contribute to reduction in torture incidents and in extreme conflicts.

In 2002, World Health Organization (WHO) passed its first Gender Policy, acknowledging the gender issue as important on its own. At about the same time, WHO began using the UN's Millennium Development Goals (MDGs), which go beyond the Health for All frameworks' focus on equity in general. They, specify, more particularly, that gender equality and the empowerment of women are vital goal.

It is important to reframe our understanding of the potential role of GD as a type III traumatic stresses and as a mediating and moderating variable of the effects of other lesser variant stressors in helping to assess and counsel females. Such paradigm shift in understanding GD will help providing more gender competent counseling for girls and women.

While explanations for the findings in this study warrant further investigation, it is clear that refugee women who have experienced significant traumatic events, particularly those who have experienced gender discrimination, are in need of interventions and supports that are different than those developed 
largely for military men suffering post-combat PTSD. Therapy for women must include a focus on empowerment, self-efficacy, and a sense of control in their new environments. The significance of these results for assessing and treating females, and especially female refugees who are either primary or secondary torture survivors is important. Assessing GD in female clients, their gender esteem, and the interaction of GD with the current other traumas is important when working with female clients. Assessment of trauma and intervention with women should address gender discrimination in counseling and therapy to re-empower victims and minimize the effects of such ongoing trauma across cultures.

Cumulative trauma complex dynamics, in GD trauma with refugees, may be better addressed effectively through parallels of multi-systemic, multi-modal, multi-component intensive interventions that match such complexity. Such multi-component interventions, addressing different levels of cognitions and affect regulation, can have synergetic effects beyond the simple added effects of their components. A wide spectrum of holistic multi-systemic, multi-modal, multi-component therapies (MSMCT), that include community healing and cultural change, have been proposed to better address such complex syndromes (e.g., Henggeler et al.,1998; Saxe, Ellis, \& Kaplow, 2007; Kira, 2002, 2010; Kira et al., 2003, 2005; Courtois , Ford, Herman, \& van der Kolk, 2009). Community-based intervenetions which go beyond home-based family sessions have shown evidence to enhance generalizabilty and durability of treatment benefits (Kazdin \& Weisz, 1998).

\section{REFERENCES}

Arbuckle, J. L. (2006). Amos 7.0 user's guide. Chicago: SPSS.

Astbury J. (2006). Gender and mental health. Paper presented under the Global Health Equity Initiative (GHEI) project on "Gender and Health Equity" based at the Harvard Center for Population and Development Studies. URL (last checked 27 August 2009). www.grhf.harvard.edu/HUpapers/gender/astbury.pdf

Astbury, J. (1999). Gender and mental health. Working Paper Series No. 99. Cambridge, MA: Harvard Center for Population and Development Studies. 8.

Berg, S. (2006). Everyday sexism and posttraumatic stress disorder in women: A correlational study. Violence Against Women, 12, 970988. doi:10.1177/1077801206293082

Bergvall, V. (1999). Toward a comprehensive theory of language and gender. Language in society, 28, 273-293. doi:10.1017/S0047404599002080

Blake, D. D., Weathers, F. W., Nagy, L. N., Kaloupek, D. G., Klauminser, G., Charney, D. S., \& Keane, T. M. (1990). A clinician rating scale for assessing current and lifetime PTSD: The CAPS-1. Behavior Therapist, 18, 187-188.

Bleier, R (1984). Science and gender: A critique of biology and its theories on women. Oxford: Pergamon.

Bruchon-Schweitzer, M. (2002). Health psychology. Paris: Edition Dunod.

Brownmiller, S. (1975). Against our will: Men, women and rape. New York: Fawcett Columbine.

Cassiman, S. A. (2005). Toward a more inclusive poverty knowledge: Traumatological contributions to the poverty discourse. The Social Policy Journal, 4, 93-106. doi:10.1300/J185v04n03 06

Christiana, E. \& Okojie, E. (1994). Gender inequalities of health in the third world. Social Science Medicine, 39, 1237-1247. doi:10.1016/0277-9536(94)90356-5

Courtois, C., Ford, J., Herman, J., van der Kolk, B. (2009). Treating complex traumatic stress disorders: An evidence-based guide. New York: Guilford Press.

Dambrun, M. (2007). Gender differences in mental health: The mediating role of perceived personal discrimination. Journal of Applied Social Psychology, 37, 1118-1129. doi:10.1111/j.1559-1816.2007.00202.x

Erceg-Hurn, D., \& Mirosevich, V. (2008). Modern robust statistical methods: An easy way to maximize the accuracy and power of your research. American Psychologist, 63, 591-601. doi:10.1037/0003-066X.63.7.591

Foley, S., Hang-Yue, N., \& Wong, A. (2005). Perceptions of Discrimination and justice: Are there Gender Differences in Outcomes? Group Organization Management, 30, 421-450. doi: $10.1177 / 1059601104265054$

Glick, P., \& Fiske, S. T. (1996). The ambivalent sexism inventory: Differentiating hostile and benevolent sexism. Journal of Personality and Social Psychology, 70, 491-512. doi:10.1037/0022-3514.70.3.491

Goldberg, D., \& Williams, P. (1988). A user's guide to the General Health Questionnaire. Windsor: NFER.

Greenberg, J. (1990). Organizational justice: Yesterday, today, and tomorrow. Journal of Management, 16, 399-432. doi: $10.1177 / 014920639001600208$

Gutek, B. A., Cohen, A. G., \& Tsui, A. (1996). Reactions to perceived discrimination. Human Relations, 49, 791-813. doi:10.1177/001872679604900604

Hawton, K., Rodham, K., Evans, E., \& Weatherall, R. (2002). Deliberate self harm in adolescents: Self report survey in schools in England. British Medical Journal, 23, 1207-1211. doi:10.1136/bmj. 325.7374 .1207

Heise, L. (1998). Violence against women: An integrated, ecological framework. Violence Against Women, 4, 262-290. doi:10.1177/1077801298004003002

Heise, L., Pitanguy, J., \& Germain, A. (1998). Violence against women: The hidden health burden. Washington DC: World Bank.

Henggeler, S. W., Schoenwald, S., Borduin, C. Rowland, M., \& Cunningham, P. (1998). Multi-systemic treatment for antisocial behavior in children and adolescents. New York: Guilford.

Hu, L., Bentler, P. M. (1999). Cutoff criteria for fit indexes in covariance structure analysis: Conventional versus new alternatives. Structural Equation Modeling, 6, 1-55. doi:10.1080/10705519909540118

Hunnicutt, G. (2009). Varieties of patriarchy and violence against women: Resurrecting "patriarchy" as a theoretical tool. Violence Against Women,15, 553-573. doi:10.1177/1077801208331246

Jenkins, R. (1991). Demographic aspects of stress. In C. L. Cooper, \& S. V. Kasl (Series Eds.), C. L. Cooper, \& R. Payne (Vol. Eds.), Personality and stress: Individual differences in the stress process (Vol. 14, pp. 107-132). Chichester: John Wiley \& Sons.

Kazdin, A. E., \& J. R. Weisz (1998). Identifying and developing empirically supported child and adolescent treatments. Journal of Counseling and Clinical Psychology, 66, 19-36. doi:10.1037/0022-006X.66.1.19

Kira, I. (2010). Etiology and Treatments of post-cumulative traumatic stress disorders in different cultures. Traumatology: An International Journal, 16, 128-141.

Kira, I. (2004). Cumulative trauma disorder: A new scale for complex PTSD. 28th International Congress of Psychology, Beijing, 8-13 August 2004

Kira, I. (2002). Torture assessment and treatment: The wraparound approach. Traumatology: An International Journal, 8, 23-51.

Kira, I. (2001). A taxonomy of trauma and trauma assessment. Traumatology: An International Journal, 2, 1-14.

Kira, I., Templin, T., Lewandowski, L., Ramaswamy, V., Bulent, O., Abu-Mediane, S., Mohanesh, J., \& Alamia, H. (2011). Cumulative tertiary appraisal of traumatic events across cultures: Two studies. Journal of Loss and Trauma: International Perspectives on Stress \& Coping, 16, 43-66.

Kira, I., Smith, I., Lewandowski, L., \& Templin, T. (2010). The effects of perceived gender discrimination on refugee torture survivors: A cross-cultural traumatology perspective. Journal of the American Psychiatric Nurses Association, 16, 299-306. doi:10.1177/1078390310384401 
Kira, I., Lewandowsk, L., Templin, T., Ramaswamy, V., Ozkan, B., \& Mohanesh, J. (2008a). Measuring cumulative trauma dose, types and profiles using a development-based taxonomy of trauma, Traumatology: An International Journal, 14, 62-87.

Kira, I., Lewandowski, L., Templin, T., Ramaswamy, V., Ozkan, B., \& Mohanesh, J. (2008b). Measuring Cumulative Trauma dose, types and profiles using a development-based taxonomy of traumas: The long version. American Psychological Association 116th Annual Convention, Boston: APA PsycExtra Data base.

Kira, I., Hammad, A., Lewandowski, L., Templin, T., Ramswamy, V., Ozkan, B., \& Mohanesh, J.(2007). The health and mental status of Iraqi refugees and their etiology. Ethnicity \& Disease, 17, 79-82.

Kira, I., Templin, T., Lewandowski, L., Clifford, D., Wiencek, E., Hammad, A., Al-Haidar, A., \& Mohanesh, J. (2006). The effects of torture: Two community studies. Peace and Conflict: Journal of Peace Psychology, 12, 205-228. doi:10.1207/s15327949pac1203 1

Kira, I., El-gouhari, G., Bazzi, H., \& Kazak, A. (2005). Treating children victims of cumulative trauma: An Innovative model. 15th National Conference on Child Abuse \& Neglect, Boston, 18-23 April 2005 .

Kira, I., Clifford, D., \& Al-Haider, D. (2003). Assessing and treating cumulative trauma disorders (CTD) in Iraqi refugees. American Psychological Association Annual Convention, Toronto, 7-11 August 2003.

Kline, R. B. (2005). Principles and practice of structural equation modeling (2nd ed.). New York: The Guilford Press.

Klonoff, E. A., Landrine, H., \& Campbell, R. (2000). Sexist discrimination may account for well-known gender differences in psychiatric symptoms. Psychology of Women Quarterly, 24, 93-99. doi:10.1111/j.1471-6402.2000.tb01025.x

Krendl, A., Richeson, J. A., Kelley, W., \& Heatherton, T. F. (2008). The negative consequence of threat: An fMRI investigation of the neural mechanisms underlying women's underperformance in math. Psychological Science, 19, 186-175.

Landrine, H., Klonoff, E. A., Gibbs, J., Manning, V., \& Lund, M. (1995). Physical and psychiatric correlates of gender discrimination: An application of the Schedule of Sexist Events. Psychology of Women Quarterly, 19, 473-492. doi:10.1111/j.1471-6402.1995.tb00087.x

Linzer, M., Spitzer, R., Kroenke, K., et al. (1996). Gender, quality of life, and mental disorders in primary care: results from the PRIMEMD 1000 study. American Journal of Medicine, 1, 526-533. doi:10.1016/S0002-9343(96)00275-6

Macintyre, S., Ford, G., \& Hunt, K. (1999). Do women "over-report" morbidity? Men's and women's responses to structured prompting on a standard question on longstanding illness. Social Science and Medicine, 48, 89-98. doi:10.1016/S0277-9536(98)00292-5

Mejía, X. E., (2005). Gender matters: Working with adult male survivors of trauma. Journal of Counseling \& Development, 83, 29-40. doi:10.1002/j.1556-6678.2005.tb00577.x

Parker G, \& Roy K. (2001) Adolescent depression: A review. Australian and New Zealand Journal of Psychiatry, 35, 572-580. doi: $10.1080 / 0004867010060504$

Petrill, S., Kovas, Y., Hart, S., Thomson, L., \& Plomin, R. (2009). The genetic and environmental etiology of high math performance in 10-years old twins. Behavior Genetics, 39, 371-379. doi:10.1007/s10519-009-9258-z

Pittaway, E. (1999). Refugee women-The unsung heroes. In B. Ferguson, \& E. Pittaway (Eds.), Nobody wants to talk about it- refugee women's mental health (Chapter 1). Sydney: Transcultural Mental Health Centre.

Pittaway, E., \& Bartolomei, L. (2001). Refugees, race, and gender: The multiple discrimination against refugee women. Refuge: Canada's Periodical on Refugees, 19, 21-32.

Richmond, J., Elliot, A., Pierce, T., Aspelmeier, J., \& Alexander, A. (2009). Polyvictimization, childhood victimization, and psychological distress in college women. Child Maltreatment, 14, 127. doi: $10.1177 / 1077559508326357$

Richeson, J. A., Baird, A. A., Gordon, H. L., Heatherton, T. F., Wyland, C. L., Trawalter, S., \& Shelton, J. N. (2003). An fMRI examination of the impact of interracial contact on executive function. Nature Neuroscience, 6, 1323-1328. doi:10.1038/nn1156

Rosenfield, S. (1999). Gender and mental health: Do women have more psychopathology, men more, or both the same (and why). In A. Horwitz, \& T. Scheid (Eds.), Handbook for study of mental health. Cambridge: Cambridge University Press.

Saxe, G., Ellis, B. H., \& Kaplow, J. (2007). Collaborative treatment of traumatized children and teens: The trauma systems therapy approach. New York: Guilford Press.

Scott S. (1998). Aggressive behavior in childhood. British Medical Journal, 316, 202-206. doi:10.1136/bmj.316.7126.202

Skarlicki, D. P., \& Folger, R. (1997). Retaliation in the workplace: The roles of distributive, procedural, and interactional justice. Journal of Applied Psychology, 82, 434-443. doi:10.1037/0021-9010.82.3.434

Stark, E. (2007). Coercive control: How men entrap women in personal life. New York: Oxford University Press.

Swim, J. K., Hyers, L. L., Cohen, L. L., \& Ferguson, M. J. (2001). Everyday sexism: Evidence for its incidence, nature, and psychological impact from three daily diary studies. Journal of Social Issues, 57, 31-53. doi:10.1111/0022-4537.00200

Walby, S. (1990). Theorizing patriarchy. Cambridge: Basil Blackwell.

Wanous, J., \& Hudy, M. J. (2001). Single-item reliability: A replication and extension. Organizational Research Methods, 4, 361-375. doi: $10.1177 / 109442810144003$

Watts, C., \& Zimmerman, C. (2002). Violence against women: Global scope and magnitude. The Lancet, 359, 1232-1237. doi:10.1016/S0140-6736(02)08221-1

Weathers, F. W., Keane, T. M., \& Davidson, J. R. (2001). Clinicianadministered PTSD scale: A review of the first ten years of research. Depression and Anxiety, 13, 132-156. doi:10.1002/da.1029

Wesley, J. (2006). Considering the context of women's violence gender, lived experiences, and cumulative victimization. Feminist Criminology, 1, 303-328. doi:10.1177/1557085106293074

Wood, W., \& Eagley, A. H. (2003). A cross-cultural analysis of the behavior of women and men: Implications for the origins of sex differences. Psychological Bulletin, 128, 699-727. doi: $10.1037 / 0033-2909.128 .5 .699$

World Health Organization (2009). Gender disparities in mental health. URL (last checked 27 August 2009). www.who.int/mental_health/media/en/242.pdf

Yllo, K. A. (1993). Through a feminist lens: Gender, power and violence. In R. J. Gelles \& D. R. Loseke (Eds.), Current controversies on family violence (pp. 47-62). Newbury Park, CA: Sage.

Zimmerman, C., Hossain, M., Yun, K., Roche, B., Morison, L., \& Watts, C. (2003). Stolen smiles: A summary report on the physical and psychological health consequences of women and adolescents trafficked in Europe. London: London School of Hygiene and Tropical Medicine. 